\title{
The Impact of Glucose Load and Worse Metabolic Status Post Initiation of Peritoneal Dialysis
}

\author{
Nadiya Al Rahbi ${ }^{1}$, Issa Al Salmi, ${ }^{1,2 *}$ (D) Sadiq Abdul Baqi ${ }^{1}$, Muna Al Bulushi' and Suad Hannawi ${ }^{3}$ (iD
}

\author{
${ }^{1}$ The Renal Medicine Department, Royal Hospital, Oman \\ ${ }^{2}$ Medicine Department, Oman Medical Specialty Board, Oman \\ ${ }^{3}$ The Medicine Department, MOHAP, UAE
}

*Corresponding author: Dr. Issa Al Salmi, MD, BA, BAO, Bch, MB (Trinity College), FRCPI, MRCP (UK), FRCP, MIPH, PhD (AUS), FASN (USA), Oman Medical Specialty Board and the Royal Hospital, 23 July Street, P O Box 1331, Code 111, Muscat, Oman, Tel: 968-92709000, Fax: 968-245-99966

\section{Abstract}

Background: End-Stage Kidney Disease (ESKD) has a major effect on global health, both as a direct cause of global morbidity and mortality. Peritoneal Dialysis (PD) utilization increases gradually due to increase of patient's awareness for different types of RRT. Glucose constitutes the main component of the PD fluid utilized by the patients.

Objectives: In this study, we aim to evaluate the various demography and clinical and laboratory data before and a year after initiation of PD and determine the impact of glucose load and worse metabolic status.

Methods: This is an observational study performed at the Royal Hospital, Muscat, Oman. All demographic, clinical and laboratory data were collected prospectively by computerized medical records system called alshifa for patients on PD following at the Renal Medicine Department from on 2015 to 2017.

Results: This study recruited 64 PD patients on PD, 48.4\% of them were male and the age mean (SD) was 46.4 (1.1) years. $76.6 \%$ were on automated peritoneal dialysis. PD therapy has positive impact on certain laboratory results, but some being affected negatively. Normal hemoglobin level $(10-13 \mathrm{~g} / \mathrm{dl})$ increased from $47.6 \%$ to $78.1 \%$ of the patients. Percentage of patients with normal serum calcium improved from $58.7 \%$ to $85.2 \%$. Percentage of patients with normal serum phosphate level reached $54.7 \%$ compared with $36.5 \%$ before PD therapy, normal potassium level increased to $85.9 \%$ compared to $58.7 \%$ pre-PD, but patients had potassium value less than $3.5 \mathrm{mmol}$ on PD increased to $12.5 \%$ from $3.2 \%$ pre-PD. On other hand, $87.5 \%$ of patients on PD had low serum albumin ( $<35 \mathrm{mg}$ ) compared with
\end{abstract}

$61.9 \%$ pre-PD and normal HBA1C level reduced to $43.5 \%$ from $54.1 \%$ before starting on PD therapy. High serum triglyceride level increased to $19.4 \%$ on PD compared to $15.4 \%$ before that, high serum glucose level increased by $1.4 \%$ and high serum cholesterol increased by $1.1 \%$ on PD.

Conclusion: This study showed improvement of patients' clinical and laboratory parameters over a year period of PD therapy. Most laboratory data and blood pressure reading improved, but there was a negative effect on some of blood investigation level, such as, glycated hemoglobin and serum albumin level.

\section{Abbreviations}

PD: Peritoneal Dialysis; OS: Observational Study; E: Epidemiology; ESKD: End-Stage Kidney Disease; RRT: Renal Replacement Therapy

\section{Introduction}

The K/DOQI has defined the Chronic Kidney Disease (CKD) as kidney damage or Glomerular Filtration Rate (GFR) $<60 \mathrm{~mL} / \mathrm{min} / 1.73 \mathrm{~m}^{2}$ for 3 months or more, irrespective of cause. Kidney damage in many kidney diseases can be ascertained by the presence of albuminuria, defined as albumin-to-creatinine ratio $>30 \mathrm{mg} / \mathrm{g}$ in two of three spot urine specimens [1]. Kidney disease has a major effect on global health [2], both as a direct cause of global morbidity and mortality and as an important risk factor for Cardiovascular Disease (CVD) [3]. Cardiovascular Disease (CVD) remains the leading cause

Citation: Al Rahbi N, Al Salmi I, Baqi SA, Al Bulushi M, Hannawi S (2020) The Impact of Glucose Load and Worse Metabolic Status Post Initiation of Peritoneal Dialysis. Int Arch Endocrinol Clin Res 6:022. doi.org/10.23937/2572-407X.1510022

Accepted: December 01, 2020: Published: December 03, 2020

Copyright: @ $2020 \mathrm{Al}$ Rahbi N, et al. This is an open-access article distributed under the terms of the Creative Commons Attribution License, which permits unrestricted use, distribution, and reproduction in any medium, provided the original author and source are credited. 
of morbidity and mortality in patients with End-Stage Kidney disease (ESKD). CKD is increasingly recognized as a global public health challenge as $10 \%$ of the global population is affected [3].

The health system in Oman is quite goods is highly recognized by World Health Organization and other various agencies $[4,5]$. Through the last four decades, a sharp progression in the prevalence of people progressing to ESKD and demanding Renal Replacement Therapy (RRT) has been noticed in Oman [6-10]. The incidence of patients with kidney disease in Oman who received RRT during 1983 was very low (21 per million populations (PMP) and has been gradually increasing (75 PMP in 2001 and 120 PMP in 2013) [11].

The prevalence of patients with ESKD on RRT in 1983 was 49, which increased to 916 in 2001 and reached 2386 in 2013. By 31 December 2013, there were 2386 patients alive on RRT, of whom 1080 were living with a functioning kidney transplant (45.3\%), 1206 were receiving hemodialysis $(50.5 \%)$, and 100 were undergoing peritoneal dialysis (4.2\%) [11]. The World Health Organization ranked Oman on the $51^{\text {st }}$ position of the top world countries where CKD is the most important causes of death and reported that CKD was the $6^{\text {th }}$ major cause of death accounting for $2.97 \%$ of total deaths (18.1 per 100000 population [4-6].

Peritoneal Dialysis (PD) is a readily available simple method for filtration of excess water, clearance of uremic toxins and electrolytes balance. It has been reported to promote improved well-being and greater personal freedom than hemodialysis through less restrictive life-style changes. It has also been argued that PD therapy should be offered to all appropriate ESKD patients and it's a good option for motivated and independent patients [12]. Moreover, Peritoneal Dialysis (PD) as the initial treatment modality in patients with ESKD gives the better preservation of residual renal function and along with its potential cardiovascular benefits. There is a superior patient survival on PD, relative to hemodialysis, as was found during the first 2 years on dialysis in both the United States and Canada. Also, there are the potential advantages of PD in terms of hepatitis C prevention, anemia management and quality of life compared to hemodialysis.

According to the late Dr. Al-Marhuby H. 1998, dialysis services were started in Oman in the form of Intermittent Peritoneal Dialysis (IPD) in Al-Nahdha hospital in the capital Muscat in $1980[6,11,13]$. The first patient treated with Continuous Ambulatory Peritoneal Dialysis (CAPD) was in Royal hospital in 1992. Oman peritoneal dialysis registry in 2016 reported that, the number of patients using this modality had jumped from 60 patients in 2012 to 142 in 2015. Hence, in order to be successful with our PD program, we need to build strong strategies to improve patients' quality of care. It can be initiated by adequate pre-dialysis care, and achieved by proper monitoring of PD quality performance, training and re-training of staff and patients, implementation of international PD guidelines and by medical research and statistical studies to evaluate and improve the provided services.

In this study, we aim to evaluate the various demography and clinical, laboratory data and the impact of glucose load and worse metabolic status before and after initiation of peritoneal dialysis.

\section{Methods}

This is an observational study performed at the Royal Hospital, Muscat, Oman. All clinical and laboratory data were collected prospectively by computerized medical records system called alshifa. All patients on Peritoneal Dialysis (PD) following the Renal Medicine department and evaluated for peritoneal dialysis as part of their investigations for the period from $1^{\text {st }}$ of January 2015 to the end of December 2017 were included in the study. Patients' data including social data, standard physical examination clinical, radiological and laboratory evaluation were retrieved from the computerized system for all peritoneal dialysis patients. Laboratory investigation and blood pressure measurement collected pre peritoneal dialysis and at one-year post PD management. Serum albumin, glucose, total cholesterol, triglycerides, and lipoprotein (a)/Lp(a)/levels were measured by quantitative kinetic spectrophotometric method using a computerized autoanalyzer AXION (Menheim Boehringer, Germany) and reagents from Boehring diagnostics. CBC, UE1, LFT, Bone, serum glucose and HBA1C were collected. The level of Parathyroid Hormone (PTH) in plasma was measured by RIA method. PCR and urinalysis obtained as well. Data about Peritoneal membranes types were collected too.

Systolic (SBP) divided to systolic Normal $<120$ $\mathrm{mmHg}$ ), pre hypertension (120-139 $\mathrm{mmHg}$ ), stage 1 hypertension (140-159 $\mathrm{mmHg}$ ), stage 2 hypertension ( $\geq$ $160 \mathrm{mmHg}$ ) and diastolic BP ( $\mathrm{mmHg}$ ) divided to normal (less than $80 \mathrm{mmHg}$ ), pre hypertension $(80-89 \mathrm{mmHg}$ ), stage 1 hypertension $(90-99 \mathrm{mmHg})$, stage 2 hypertension ( $\geq 100)$.

Serum Hemoglobin level was divided into three categories; less than $(10 \mathrm{~g} / \mathrm{dl}),(10-13 \mathrm{~g} / \mathrm{dl})$ and $(>13 \mathrm{~g} / \mathrm{dl})$. Serum Hematocrit level divided into normal (35-45\%) and abnormal level. Serum cholesterol level divided to normal level $(5 \mathrm{mmoL} / \mathrm{L})$ level, border line cholesterol level (5.2-6.1 mmol/l) high cholesterol $(6.2-6.6 \mathrm{mmol} / \mathrm{l})$. Serum Triglyceride level divided to normal level (1.7 $\mathrm{mmol} . \mathrm{L})$, border line (1.7-2.2 $\mathrm{mmol} / \mathrm{L})$ and high level (> $2.2 \mathrm{mmol} / \mathrm{L}$ ). HBA1C has been classified as Normal, Pre-diabetes (42-47) and diabetic ( $\geq 48)$.

Serum albumin level classified to low $(<35 \mathrm{~g} / \mathrm{L})$, normal level $(35 \mathrm{~g} / \mathrm{dl})$ and high $(>48 \mathrm{~g} / \mathrm{L})$. Serum phosphate level classified to low $(<0.75 \mathrm{mmol} / \mathrm{l})$, normal level (0.75-1.5 mmol/L) and high level (> $1.5 \mathrm{mmol} / \mathrm{L})$. Para- 
thyroid Hormone (PTH) level divided to $<65 \mathrm{pg} / \mathrm{ml}, 65$ $\mathrm{pg} / \mathrm{ml}$ and above $65 \mathrm{pg} / \mathrm{ml}$. Serum glucose divided into three categories, low $<3 \mathrm{mmol} / \mathrm{l}$, normal (3-6.9 mmol/l) and high $>7 \mathrm{mmol} / \mathrm{l}$.

Urine analysis included specific gravity (High $>1.03$, Low $<1.02$ and Normal (1.02-1.03), urine $\mathrm{PH}$ (Acidic $<7$, normal 7 and alkaline $>7$ ).

The ages and genders of the dialysis patients were recorded. The heights and weights (dry weights) of the patients were determined by using calibrated height/ weight scales. The body mass index of each patient was calculated using the formula weight/ (height) ${ }^{2}$. The arterial blood pressure of the patients was measured three times on both arms, and the average of the last two measurements was recorded. A desktop sphygmomanometer (Erkameter 3000 with standard mercury) was used to measure the arterial blood pressure. To evaluate the biochemical parameters, venous blood samples were collected after 12 hours of fasting in the morning and before starting dialysis therapy.

Each value expressed as the mean \pm SD. $P$ values less than 0.05 considered statistically significant. All statistical calculations performed with the STATA 16 software (version 16.0, STATA Inc, Chicago, Illinois, USA).

\section{Results}

There were 64 adult patients on peritoneal dialysis therapy following at Renal Medicine Department, of which $48.4 \%$ of them were male and $51.7 \%$ were female. Their age ranged between 13 and 80 years with a mean (SD) of 46.4 (1.1) years. Of the total participants, $76.6 \%$ were on Automated Peritoneal Dialysis (APD) therapy and $23.4 \%$ were on Continues Ambulatory Peritoneal Dialysis (CAPD). The mean (SD) of BMI was 25.4 (6.9) $\mathrm{kg} / \mathrm{m}^{2}$, of which $45.3 \%$ of patients had normal weight, $15.6 \%$ of patients were under weight $\left(<18 \mathrm{~kg} / \mathrm{m}^{2}\right)$ and $39 \%$ of them had overweight ( $\left.>25 \mathrm{~kg} / \mathrm{m}^{2}\right)$, as shown in Table $1.45 .3 \%$ of total patients took care of PD by themselves and others depended on others. Moreover, out of total PD care givers, there were more than $50 \%$ had higher education, Figure 1.

Total of $48.4 \%$ of patients were diabetics, $89 \%$ were hypertensive, and $46.9 \%$ had coronary artery disease. Of the total participants, $46.9 \%$ were having other disease in addition to what is stated before. These diseases classified to urology diseases, immune disease, hematology, lung diseases and trauma. Firstly, Urology diseases such as neurogenic bladder, recurrent UTI, obstructive uropathy and Posterior Urethral Valves (PUV), Benign Prostate Hypertrophy (BPH) and kidney stone disease. Secondly, connective tissue disease such as Alport syndrome and lupus nephritis. Thirdly, there were lung diseases, for example, bronchial asthma and TB Lymphadenitis. Fourthly, hematology diseases, such as sickle cell disease and chronic anemia. Finally, trauma was reported such as leg fracture and hip fracture, Table 1.
Table 1: Shows the various demographic and participants characteristics.

\begin{tabular}{|c|c|}
\hline Variables & Mean (\%) \\
\hline \multirow[t]{2}{*}{ Gender } & Male: $48.4 \%$ \\
\hline & Female: $51.6 \%$ \\
\hline \multirow[t]{6}{*}{ Age/year } & 46.4 (1.1) years, Range: $13-81$ \\
\hline & 1. $13-20(17.2 \%)$ \\
\hline & 2. $21-40(18.8 \%)$ \\
\hline & 3. $41-60(35.9 \%)$ \\
\hline & 4. $61-80(28.1 \%)$ \\
\hline & 5. > 80 years $(0 \%)$ \\
\hline \multirow[t]{5}{*}{ Height/cm } & $157(10.6) \mathrm{cm}$, Range: $134-177$ \\
\hline & 1. $<147(23.4 \%)$ \\
\hline & 2. $148-155(15.6 \%)$ \\
\hline & 3. $156-170(43.8 \%)$ \\
\hline & 4. > $170(17.2 \%)$ \\
\hline \multirow[t]{7}{*}{ Weight/kg } & 63.8 (20.3), Range: $27-130$ \\
\hline & 1. $<46(23.4 \%)$ \\
\hline & 2. $47-54(12.5 \%)$ \\
\hline & 3. $55-65(17.2 \%)$ \\
\hline & 4. $67-80(28.1 \%)$ \\
\hline & 5. $81-95(17.2 \%)$ \\
\hline & 6. > $95(1.6 \%)$ \\
\hline \multirow[t]{4}{*}{ BMI } & 25.4 (6.9), Range: $13-24.8$ \\
\hline & 1. Underweight $<18(5.6 \%)$ \\
\hline & 2. Normal Weight $18-24.9(45.3 \%)$ \\
\hline & 3. Overweight > $25(39 \%)$ \\
\hline \multirow[t]{2}{*}{ Diabetic Mellitus } & 1. Yes: $48.4 \%$ \\
\hline & 2. No: $51.6 \%$ \\
\hline \multirow[t]{2}{*}{ Hypertension } & 1. Yes: $89.1 \%$ \\
\hline & 2. No: $10.9 \%$ \\
\hline \multirow{2}{*}{ Coronary artery } & 1. Yes: $46.9 \%$ \\
\hline & 2. No: $53.1 \%$ \\
\hline \multirow[t]{2}{*}{ Other Diseases } & 1. Yes: $46.9 \%$ \\
\hline & 2. No: $53.1 \%$ \\
\hline \multirow[t]{2}{*}{ Residual renal function } & 1. Yes: $68.8 \%$ \\
\hline & 2. No: $31.3 \%$ \\
\hline \multirow[t]{2}{*}{ PD Modality } & 1. CAPD: $23.4 \%$ \\
\hline & 2. APD: $76.6 \%$ \\
\hline Type of peritoneal & 1. Low Average: $38.5 \%$ \\
\hline \multirow[t]{3}{*}{ Membrane } & 2. Low: $11.5 \%$ \\
\hline & 3. High: $9.6 \%$ \\
\hline & 4. High average: $40.4 \%$ \\
\hline \multirow{2}{*}{$\begin{array}{l}\text { History of Hemodialysis } \\
\text { before PD }\end{array}$} & 1. Permanent HD: $45.3 \%$ \\
\hline & 2. Temporary HD: $17.2 \%$ \\
\hline \multirow{2}{*}{$\begin{array}{l}\text { History of Kidney } \\
\text { Transplantation before } \\
\text { PD }\end{array}$} & 1. Yes: $6.3 \%$ \\
\hline & 2. No: $93.7 \%$ \\
\hline Pre-emptive PD & $37.5 \%$ \\
\hline
\end{tabular}

Kidney Ultrasound Graph (USG) recorded right and left kidney length. There were $62.5 \%$ of total patients had small right kidney $(<9 \mathrm{~cm}), 31.1 \%$ had normal right kidney size $(9-14 \mathrm{~cm})$ and $6.4 \%$ of them their right kidney was enlarged $(>14 \mathrm{~cm})$, while $55.1 \%$ of participants 


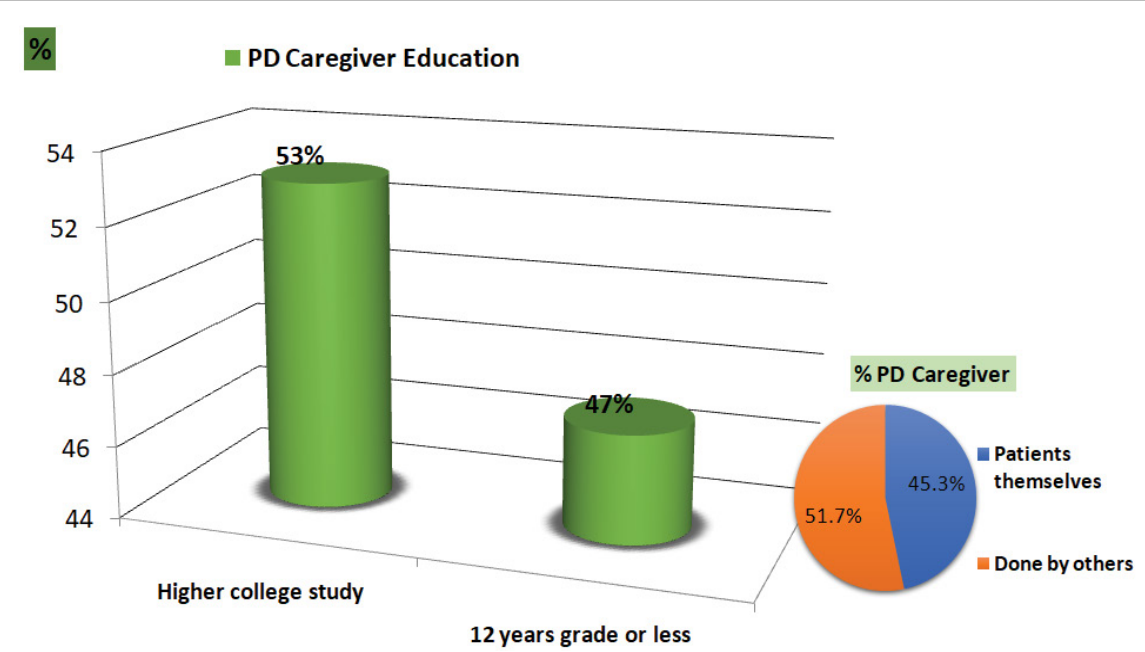

Figure 1: Shows the percentage of patients who perform dialysis themselves and those require caregiver help to perform the dialysis therapy and their education level.

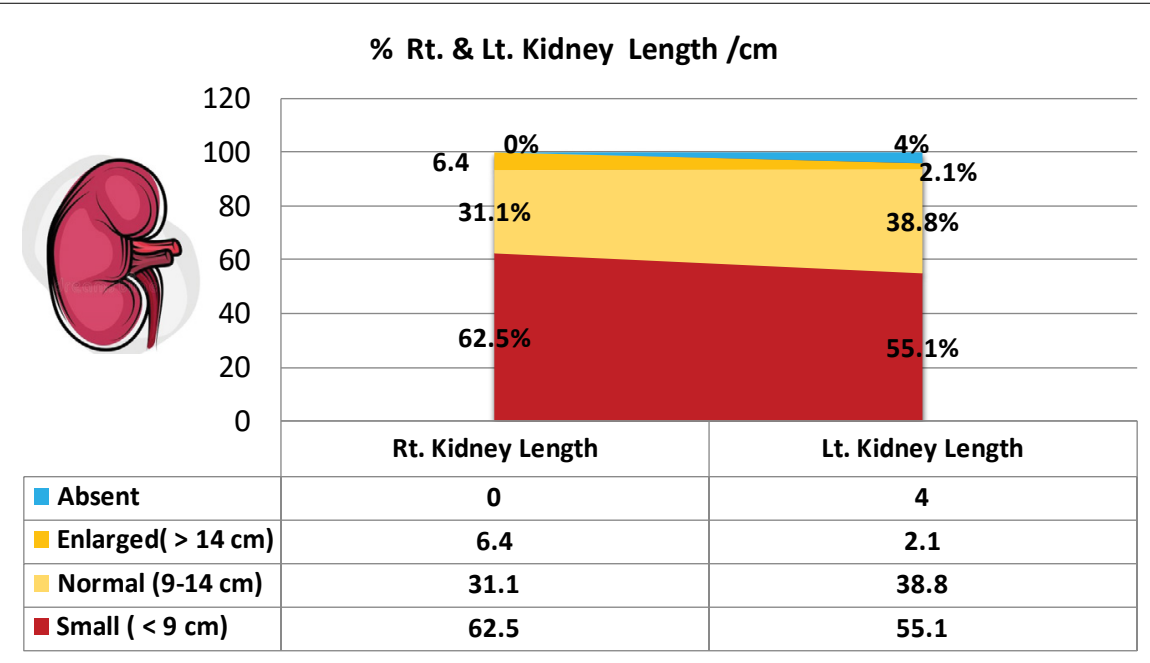

Figure 2: Shows the ultrasound measurements of kidney length.

had small left kidney, 38.8\% had normal length, 2.1\% had enlarged left kidney and $4 \%$ their left kidney was absent, Figure 2.

The percentage of patients started PD as preemptive therapy was $37.5 \%, 13.7 \%$ of them were temporary started on hemodialysis, $6.3 \%$ had failed kidney transplantation graft and $45.3 \%$ were on permanent hemodialysis and moved to PD mostly due to vascular access failure.

The majority (40.4\%) of patients were having high average membrane, followed by Low Average, low and high membrane-transporter, with a percentage of $38.5 \%, 11.5 \%$, and $9.6 \%$, respectively. PD Catheter (PDC) survival was calculated per years with the highest percentage $(70.3 \%)$ had PDC for $3-5$ years, $37.5 \%$ from 1 to 3 years and $21.9 \%$ of patients spent more than 5 years on PD.

Figure 3 showed the $24 \mathrm{hrs}$ urine collection for volume measurement $/ \mathrm{ml}$ classified to three categories $>$ $500 \mathrm{ml} /$ day, $100-500 \mathrm{ml} /$ day and $<100 \mathrm{ml} /$ day. There were 13 (64) patients their $24 \mathrm{hrs}$ urine collection more than $500 \mathrm{ml}, 28$ (64) less than $500 \mathrm{ml}$ and 23 (64) on anuria stage.

Our participants mostly depended on glucose (Dianeal) PD fluid to perform their dialysis because it is well accessible. They used different dianeal concentration depend on their fluid status and residual renal function. There were a total of 33 (49) patients on APD using two bags of $2.27 \%$ daily. $67.2 \%$ of total patients consumed about $4.5 \%$ of glucose PD fluid on daily basis. All anuric patients were given hypertonic solution (3.86\%) for emergency use and they used an average of 3-4 bags per months. $55 \%$ of total patients were on Extraneal (Icodextrin) and $4.7 \%$ of them were on Nutraneal fluid (amino acid) beside glucose fluid.

The mean (SD) of systolic blood pressure was 145 (21.2) $\mathrm{mmHg}$ at pre-initiation on PD and improved to 134 (12.8) $\mathrm{mmHg}$ post PD. The mean (SD) of diastolic blood pressure was 82 (14.6) $\mathrm{mmHg}$ at pre-PD and improved to 77 (9.3) mmHg post PD, as shown in Table 2.

There were significant improvements for most of tests' results after starting on PD, while some labs get 


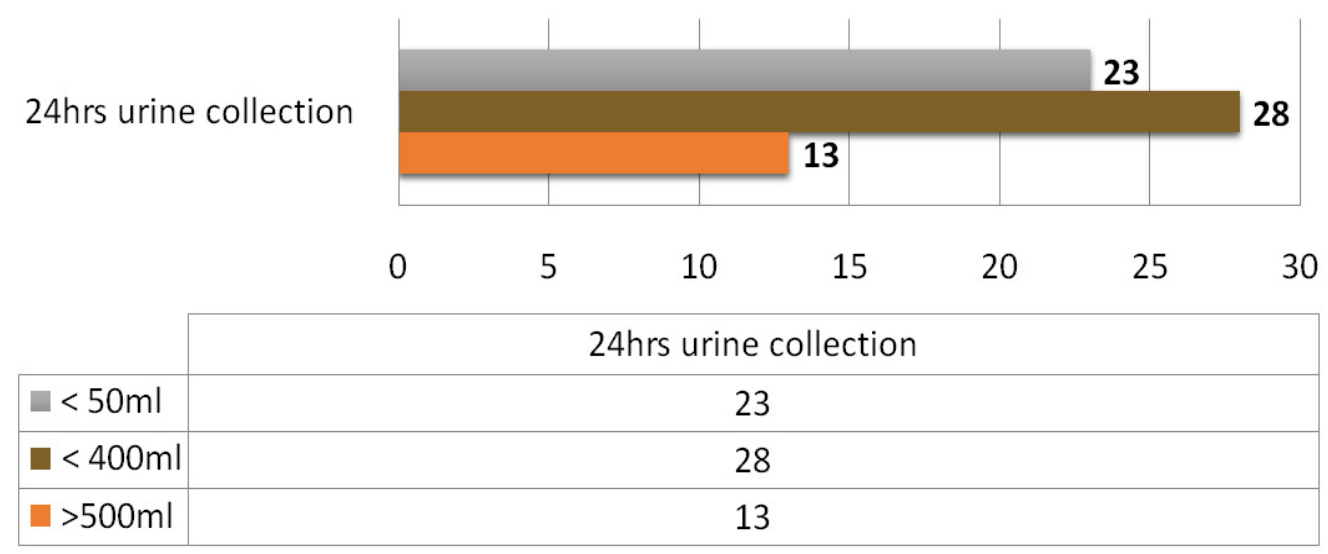

\section{$\mathrm{Ml} / 24 \mathrm{hrs}$}

Figure 3: Shows the 24 hrs urine collection among the participants.

Table 2: Shows the mean (SD) and various stages of systolic and diastolic blood pressure readings of participants before and a year after starting peritoneal dialysis.

\begin{tabular}{|c|c|c|}
\hline Variables & Pre PD & Post PD \\
\hline Systolic Blood Pressure & $\begin{array}{l}\text { 145.0 (21.2) } \mathrm{mmHg} \\
\text { 1. Systolic Normal (< 120): } 9.68 \% \\
\text { 2. Prehypertension (120-139): } 16.1 \% \\
\text { 3. Stage } 1 \text { Hypertension } 140-159: 37.1 \% \\
\text { 4. Stage } 2 \text { Hypertension }(\geq 160): 37.1 \%\end{array}$ & $\begin{array}{l}\text { 134.0 (12.8) } \mathrm{mmHg} \\
\text { 1. Systolic Normal (<120): } 10.9 \% \\
\text { 2. Pre hypertension (120-139): } 16.1 \% \\
\text { 3. Stage } 1 \text { Hypertension: } 42.2 \% \\
\text { 4. Stage } 2 \text { Hypertension: } 1.6 \%\end{array}$ \\
\hline Diastolic Blood pressure & $\begin{array}{l}\text { 82.0 (14.6) } \mathrm{mmHg} \\
\text { 1. Normal (less than } 80): 41.9 \% \\
\text { 2. Prehypertension ( } 80-89): 21 \% \\
\text { 3.Stage } 1 \text { Hypertension (90-99): } 19.3 \% \\
\text { 4. Stage } 2 \text { Hypertension ( } \geq 100): 17.7 \%\end{array}$ & $\begin{array}{l}77.0(9.3) \mathrm{mmHg} \\
\text { 1. Normal (less than } 80): 42.2 \% \\
\text { 2. Prehypertension ( } 80-89): 43.8 \% \\
\text { 3. Stage } 1 \text { Hypertension }(90-99): 12.5 \% \\
\text { 4. Stage } 2 \text { Hypertension }(\geq 100): 1.6 \%\end{array}$ \\
\hline
\end{tabular}

Table 3: Shows the mean (SD) and percentages of categorization of various Laboratory tests before and a year after starting peritoneal dialysis.

\begin{tabular}{|c|c|c|}
\hline Variables & Pre PD & Post PD \\
\hline HBA $1 \mathrm{C} \%$ & $\begin{array}{l}44(19.4) \\
\text { 1. Normal (42): } 54.1 \% \\
\text { 2. Prediabetes }(42-47): 10.8 \% \\
\text { 3. Diabetic (> 48): } 35.1 \%\end{array}$ & $\begin{array}{l}52(25.7) \\
\text { 1. Normal }(42): 43.5 \% \\
\text { 2. Pre diabetes }(42-47): 8.7 \% \\
\text { 3. Diabetic }(>48): 47.8 \%\end{array}$ \\
\hline Hemoglobin $(\mathrm{Hb}) \mathrm{g} / \mathrm{dl}$ & $\begin{array}{l}\text { 10.3 (1.8) } \\
\text { 1. }<10: 47.6 \% \\
\text { 2. } 10-13: 46 \% \\
\text { 3. }>13: 6.4 \%\end{array}$ & $\begin{array}{l}11(1.3) \\
\text { 1. < 10: } 15.6 \% \\
\text { 2. } 10-13: 78.1 \% \\
\text { 3. > 13: } 6.3 \%\end{array}$ \\
\hline Hematocrit & $\begin{array}{l}31.3(6) \\
\text { 1. Normal }(35-45 \%): 25.4 \% \\
\text { 2. Abnormal: } 74.6 \%\end{array}$ & $\begin{array}{l}\text { 33.4 (5.5) } \\
\text { 1. Normal }(35-45 \%): 43.8 \% \\
\text { 2. Abnormal: } 56.3 \%\end{array}$ \\
\hline Serum Red Blood Cells (RBC) & $\begin{array}{l}\text { 4.0 }(0.9) \\
\text { 1. Low }(<4.1): 49.2 \% \\
\text { 2. Normal }(4.1-5.4): 38.1 \% \\
\text { 3. High }(>5.4): 12.7 \%\end{array}$ & $\begin{array}{l}\text { 4.8 (4.5) } \\
\text { 1. Low: } 34.4 \% \\
\text { 2. Normal }(4.1-5.4): 60.9 \% \\
\text { 3. High: } 4.7 \%\end{array}$ \\
\hline Serum platelet & $\begin{array}{l}248(83.8) \\
\text { 1. Normal (150-450): } 93.7 \% \\
\text { 2. Abnormal: } 6.4 \%\end{array}$ & $\begin{array}{l}253.9(74.2) \\
\text { 1. Normal (150-450): } 95.3 \% \\
\text { 2. Abnormal: } 4.7 \%\end{array}$ \\
\hline Serum white blood count & $\begin{array}{l}6.7(2) \\
\text { 1. Normal }(2.2-10): 93.7 \% \\
\text { 2. Abnormal: } 6.4 \%\end{array}$ & $\begin{array}{l}\text { 6.6 }(2.2) \\
\text { 1. Normal }(2.2-10): 95.3 \% \\
\text { 2. Abnormal: } 4.7 \%\end{array}$ \\
\hline
\end{tabular}




\begin{tabular}{|c|c|c|}
\hline Serum Sodium & $\begin{array}{l}\text { 135.8 (3.7) } \\
\text { 1. Normal (135-145): } 61.9 \% \\
\text { 2. Abnormal: } 38.1 \%\end{array}$ & $\begin{array}{l}\text { 137.0 (2.7) } \\
\text { 1. Normal (135-145): } 84 \% \\
\text { 2. Abnormal: } 15.6 \%\end{array}$ \\
\hline Serum potassium & $\begin{array}{l}4.9(1.0) \\
\text { 1. Normal }(3.5-5): 58.7 \% \\
\text { 2. High }(>5): 38.1 \% \\
\text { 3. Low }(<3.5): 3.2 \%\end{array}$ & $\begin{array}{l}4.0(0.6) \\
\text { 1. Normal }(3.5-5): 85.9 \% \\
\text { 2. High: } 1.6 \% \\
\text { 3. Low: } 12.5 \%\end{array}$ \\
\hline Carbon Dioxide $\left(\mathrm{CO}_{2}\right)$ & $\begin{array}{l}\text { 17.0 (5) } \\
\text { 1. Normal }(22-29): 12.7 \% \\
\text { 2. Low: } 87.3 \% \\
\text { 3. High: } 0 \%\end{array}$ & $\begin{array}{l}22.2(2.5) \\
\text { 1. Normal }(22-29): 64.1 \% \\
\text { 2. Low: } 35.9 \% \\
\text { 3. High: } 0 \%\end{array}$ \\
\hline Serum urea & $\begin{array}{l}28.5(11) \\
\text { 1. less than } 17 \mathrm{mmol} / \mathrm{l}: 14.3 \% \\
\text { 2. (17-33) } \mathrm{mmol} / \mathrm{L}: 58.7 \% \\
\text { 3. More than } 33 \mathrm{mmol} / \mathrm{L}: 27 \%\end{array}$ & $\begin{array}{l}\text { 18.8 (6.7) } \\
\text { 1. less than } 17 \mathrm{mmol} / \mathrm{l}: 45.3 \% \\
\text { 2. (17-33): } 54.7 \% \\
\text { 3. More than } 33: 0 \%\end{array}$ \\
\hline Serum Creatinine & $\begin{array}{l}832.5(296.8) \\
\text { 1. } 300-500: 9.5 \% \\
\text { 2. } 500-1000: 65.1 \% \\
\text { 3. > 1000: } 25.4 \%\end{array}$ & $\begin{array}{l}792.7(284.6) \\
\text { 1. } 300-500: 21.9 \% \\
\text { 2. } 500-1000: 64.1 \% \\
\text { 3. > 1000: } 14.1 \%\end{array}$ \\
\hline Glomular Filtration Rate (GFR) ml/min/1.7 & $\begin{array}{l}6.7(2.5) \\
1 .>15: 0 \% \\
\text { 2. } 14-10: 7 \% \\
\text { 3. } 10-5: 73.7 \% \\
4 .<5: 19.3 \%\end{array}$ & $\begin{array}{l}7.2(3.3) \\
\text { 1. > 15: } 3.3 \% \\
\text { 2. } 14-10: 13.3 \% \\
\text { 3. } 10-5: 65.1 \% \\
\text { 4. < } 5: 18.3 \%\end{array}$ \\
\hline Serum calcium (mg) & $\begin{array}{l}\text { 2.4 }(0.3) \\
\text { 1. Low }(<2.1): 17.5 \% \\
\text { 2. Normal }(2.1-2.6): 58.7 \% \\
\text { 3. High }(>2.6): 23.8 \%\end{array}$ & $\begin{array}{l}\text { 2.4 }(0.2) \\
\text { 1. Low }(<2.1: 1.6 \% \\
\text { 2. Normal }(2.1-2.6): 82.8 \% \\
\text { 3. High }(>2.6): 15.6 \%\end{array}$ \\
\hline Serum phosphate & $\begin{array}{l}\text { 1.5 }(0.6) \\
\text { 1. Low }(<0.75): 2 \% \\
\text { 2. Normal }(0.75-1.5): 36.5 \% \\
\text { 3. High (> 1.5): } 61.5\end{array}$ & $\begin{array}{l}\text { 1.8 (0.5) } \\
\text { 1. Low: } 0 \\
\text { 2. Normal }(0.75-1.5): 54.7 \% \\
\text { 3. High: } 45.3 \%\end{array}$ \\
\hline Serum Albumin & $\begin{array}{l}\text { 31 (5.6) } \\
\text { 1. Low: } 61.9 \% \\
\text { 2. Normal }(35-50 \mathrm{~g} / \mathrm{l}): 38.1 \% \\
\text { 3. High: } 0\end{array}$ & $\begin{array}{l}29.3(4.5) \\
\text { 1. Low: } 87.5 \% \\
\text { 2. Normal }(35-50 \mathrm{~g} / \mathrm{l}): 12.5 \% \\
\text { 3. High: } 0\end{array}$ \\
\hline Parathyroid Hormone PTH pg/ml & $\begin{array}{l}57.9(48.1) \\
\text { 1. less than } 65 \mathrm{pg} / \mathrm{m}: 68.9 \% \\
\text { 2. } 65 \mathrm{pg} / \mathrm{m}: 1.6 \% \\
\text { 3. Above } 65: 29.5 \%\end{array}$ & $\begin{array}{l}59.5(56.9) \\
\text { 1. less than } 65 \mathrm{pg} / \mathrm{m}: 72.9 \% \\
\text { 2. } 65 \mathrm{pg} / \mathrm{m}: 1.7 \% \\
\text { 3. Above } 65: 25.4 \%\end{array}$ \\
\hline serum glucose & $\begin{array}{l}\text { 7.4 }(7.4) \\
\text { 1. Low }(<3) \\
\text { 2. Normal }(3-6.9): 64.3 \% \\
\text { 3. High }(>7): 35.7 \%\end{array}$ & $\begin{array}{l}\text { 2.4 (6.5) } \\
\text { 1. Low: } 0 \\
\text { 2. Normal }(3-6.9): 62.9 \% \\
\text { 3. High } 37.1 \%\end{array}$ \\
\hline serum total cholesterol & $\begin{array}{l}\text { 4.5 (1.5) } \\
\text { 1. Normal }(5 \mathrm{mmoL} / \mathrm{L}): 71.4 \% \\
\text { 2. Borderline: } 16.3 \% \\
\text { 3. High (5.2-6.6): } 12.2 \%\end{array}$ & $\begin{array}{l}\text { 4.7 (1.4) } \\
\text { 1. Normal ( } 5 \mathrm{mmoL} / \mathrm{L}): 73.3 \% \\
\text { 2. Borderline: } 13.3 \% \\
\text { 3. High (5.2-6.6): } 13.3 \%\end{array}$ \\
\hline $\begin{array}{l}\text { Serum Triglycerides } \\
\text { Mmol/l }\end{array}$ & $\begin{array}{l}\text { 1.6 }(0.7) \\
\text { 1. Normal (1.7MMOL/L): } 64.1 \% \\
\text { 2. Borderline high (1.7-2.2): } 20.5 \% \\
\text { 3. High: } 15.4 \%\end{array}$ & $\begin{array}{l}\text { 1.7 }(0.9) \\
\text { 1. Normal }(1.7 \mathrm{mmol} / \mathrm{L}): 58.3 \% \\
\text { 2. Borderline high (1.7-2.2): } 22.2 \% \\
\text { 3. High: } 19.44 \%\end{array}$ \\
\hline Low density cholesterol/mmol/I & $3(1.1)$ & $2.6(1.2)$ \\
\hline
\end{tabular}


worsen on PD, as shown in Table 3. Normal hemoglobin $(\mathrm{Hb})(10-13 \mathrm{~g} / \mathrm{dl})$ was $46 \%$ of total patients then the percentage improved post PD to $78.1 \%$. The mean (SD) of Hematocrit was 31.3 (6) before starting on PD, while it increased to 33.4 (5.5) post-PD. The percentage of patients having normal potassium level $(3.5-6 \mathrm{mmol} / \mathrm{l})$ post-PD increased to $85.9 \%$ compared to $58.7 \%$ pre-PD and the percentage of hyperkalemia Figure 4 . The mean (SD) of serum sodium level was 135.8 (3.7), which improved to 137 (2.7) post PD. Acidosis improved post-PD by about $49 \%$. Serum calcium and phosphate had positive effect post PD, there were $17.5 \%$ of patients had low calcium before starting on PD, then the percentage of them reduced to $1.6 \%$ only, and $85.2 \%$ had normal calcium value post PD compared with $58.7 \%$ pre-PD. The percentage of normal serum phosphate level reached to $54.7 \%$ compared with $36.5 \%$ before PD.

On other hand, some variables been affected negatively by peritoneal dialysis initiation, as shown in graphs 5 and 6 . Firstly, HBA1C has been classified as Normal, Pre-diabetes (42-47) and diabetic $(\geq 48)$. Normal HBA1C level reduced to $43.5 \%$ from $54.1 \%$ of patients before starting on PD, and diabetic cases went up to $47.8 \%$ from $35.1 \%$ of patients, as shown in Figure 5.

Another variable been disturbed post PD is serum albumin, our finding showed that, patients on peritoneal dialysis were losing albumin, about $87.5 \%$ of patients on PD had low serum albumin $(<35 \mathrm{~g})$ compared with $61.9 \%$ pre PD. Normal level of serum Albumin (35-40g) was $38.1 \%$ pre PD then it reduced to $12.5 \%$, as shown in Figure 6.

The percentage of patients with high serum triglyceride pre-initiation on PD was $15.4 \%$ then it increased to $19.4 \%$ on PD. Moreover, the percentage of patients having high serum glucose level increased by $1.4 \%$ and high serum cholesterol increased by $1.1 \%$ on PD, Table 3.

Regarding urine analysis, $40 \%$ and $37.5 \%$ of patients had positive leucocytes in their urine at pre-PD and post-PD, respectively. Only $4.9 \%$ of patients had nitrate pre-PD and $8.6 \%$ post-PD. $95.2 \%$ of patients had positive protein in urine pre-PD and remained high post$\mathrm{PD}$ at $87.5 \%$. The percentage of glucose in urine was $58.5 \%$ pre-PD and almost the same post-PD of $59.4 \%$.

\section{Serum Potassium \%}

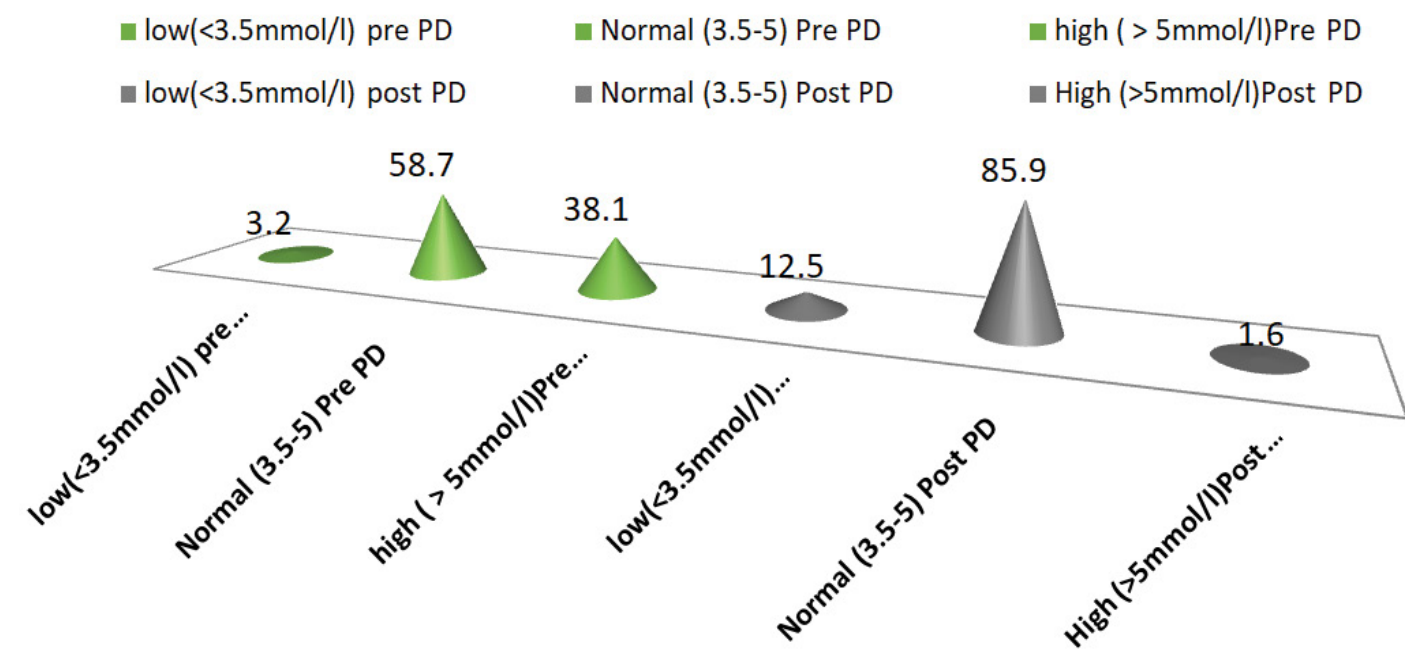

Figure 4: Shows the percentage of serum potassium levels before and post Peritoneal Dialysis (PD).

\% HBA1C Pre PD

\% HBA1C Post PD

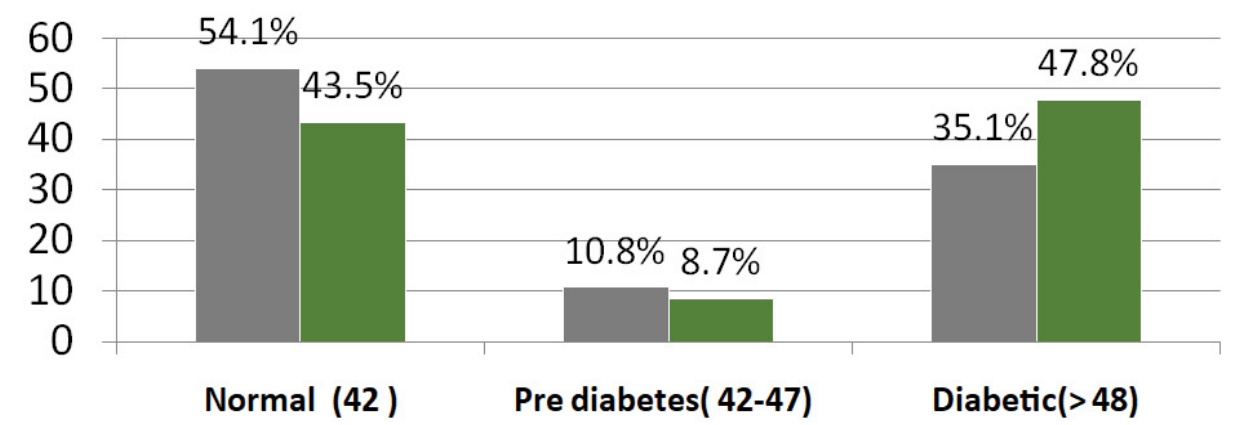

Figure 5: Shows the glycated hemoglobulin (HBA1C) levels before and after starting on Peritoneal Dialysis (PD). 


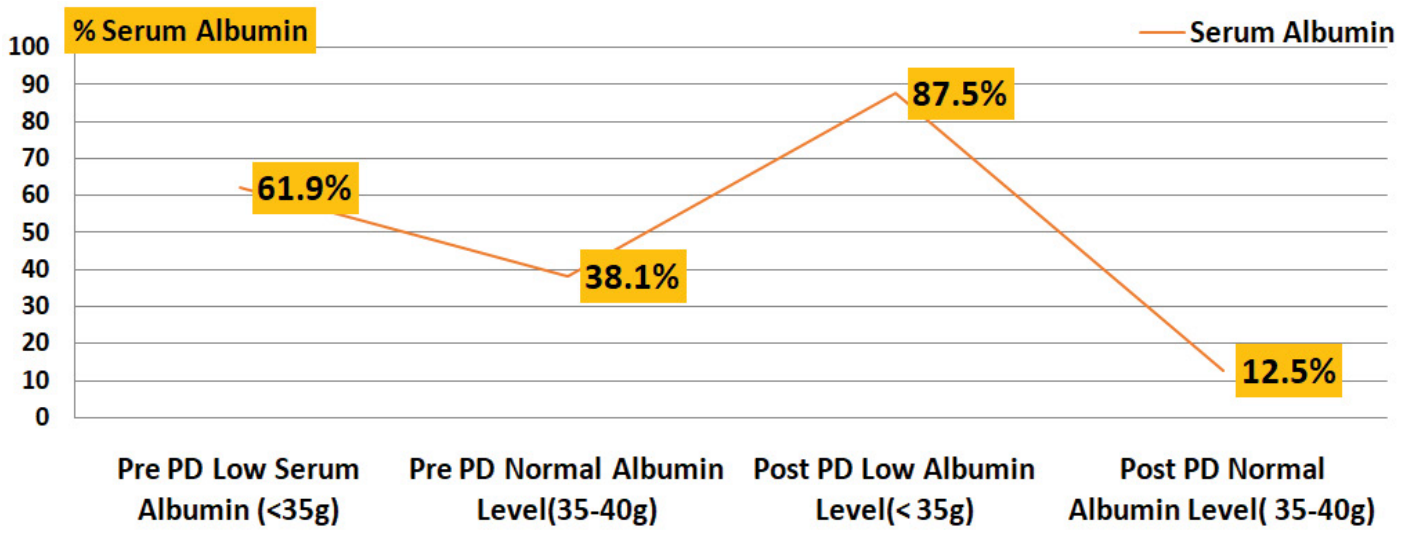

Figure 6: Shows the percentage of serum albumin levels of patients at pre and post Peritoneal Dialysis (PD).

Ketone in urine increased post-PD to $12.5 \%$ compared with $4.9 \%$ during pre-PD. Bilirubin in urine remained negative before and after starting on PD. Blood in Urine reduced by $19.5 \%$ post-PD, the percentage was 97.6 Pre PD and 78.1 post PD.

\section{Discussion}

This study evaluated the clinical and laboratory data of patients on peritoneal dialysis following with the Renal Medicine department at Royal Hospital before and a year after peritoneal dialysis. It analyzed the patients' data including standard clinical examination and laboratory evaluation and the impact of glucose load and worse metabolic status before and after initiation of peritoneal dialysis. The population was composed of almost equal gender distribution with a mean age of 46.4 years. Of note, the total participants, $76.6 \%$ were on Automated Peritoneal Dialysis (APD) therapy. There were significant improvements for the first year period on PD for most patients and these improvements include laboratory parameters and blood pressure control. However, some parameters been affected with PD negatively such as serum albumin and $\mathrm{HbA} 1 \mathrm{C}$.

The participants mean age was 46.4 (1.1) years which is similar to Iranian PD patients' age, where their mean age was 46 years [14] and it is very close to Saudi PD patients in a study done at King Khalid University Hospital [15]. The number of female patients were slightly higher than male patients, females were $51.7 \%$ and males were $48.4 \%$. Saudi male PD patients were $46 \%$ and Iranian male patients were $55 \%$. The study of Al Rahbi N 2020 had close finding were the main age for PD patients was $41.7 \pm 24.3$ and the percentage of female were $60.4 \%$ [16].

Body Mass Index (BMI) was classified into three categories: underweight $(<18.5) \mathrm{kg} / \mathrm{m}^{2}$, normal weight $(18.5$ $\left.24.9 \mathrm{~kg} / \mathrm{m}^{2}\right)$ and overweight $\left(>25 \mathrm{~kg} / \mathrm{m}^{2}\right)$. The mean BMI was 25.4 (6.9), of which $45.3 \%$ of patients had normal weight, $15.6 \%$ of patients were under weight and $39 \%$ of them had overweight. The minimum BMI was $13 \mathrm{~kg} /$ $\mathrm{m}^{2}$ and the maximum BMI was $47.8 \mathrm{~kg} / \mathrm{m}^{2}$. An Indian study [17] found that of the 328 PD patients, 47 (14.3\%) were underweight, 171 (52.1\%) were normal weight, 53 (16.2\%) were overweight, and 57 (17.4\%) were obese at commencement of PD therapy with mean BMI: $21.9 \pm$ $3.8 \mathrm{~kg} / \mathrm{m}^{2}$ ) for $20.0 \pm 14.3$ months. Another study done in Hong Kong for 274 PD patient, found that the mean BMI was $21.97 \pm 3.23 \mathrm{~kg} / \mathrm{m}^{2}$; 37 underweight (13.5\%); 35 obese (12.8\%) [18].

The present study found that $48.4 \%$ of patients were diabetics, $89 \%$ were hypertensive, and $46.9 \%$ had coronary artery disease. A $46.9 \%$ of total patients were having other disease beside the previous diseases. Researchers found that over 50 to $60 \%$ of hemodialysis patients and nearly $30 \%$ PD patients are hypertensive $[14,19,20]$. Blood pressure measurement classified to four categories: Systolic normal $(<120)$, pre-hypertension (120-139), stage 1 Hypertension and stage $2 \mathrm{Hy}-$ pertension. The mean systolic blood pressure was 145 (21.2) at pre initiation on PD and improved to134 (12.8) post PD. Mean Diastolic BP at pre PD was 82 (14.6) and improved to 77 (9.3) post PD. Daily PD therapy along with residual renal function and salt diet restriction made PD effective treatment to control blood pressure, some of hypertensive patients on PD patients achieved reduced the dose of antihypertensive medication and some of them even able to hold their antihypertensive medication for the first period on PD. Peritoneal dialysis is a daily therapy, so it may not be surprising that only 30 percent of patients are estimated to require hypertension therapy. However, excess fluid in the body still plays a major role in causing the blood pressure to be high, and many peritoneal dialysis patients do not keep the fluid off as well as they should $[19,20]$.

In the present study, about 41 (64\%) of total patients were still having good Residual Renal Function (RRF). It is well known that the preservation of residual renal function makes a big difference in solute clearance and control fluid status and there are many studies support such a view including B Marro, et al. 2008 [21] and Roszkowska-Blaim, et al. 2013 [22]. Marro, et al. 2008 had mention that preservation of RRF is associated with better long-term survival (lower relative risk of death) in dialysis patients, $11-17 \mathrm{mmHg}$ reduction in Blood Pres- 
sure (BP), $18 \mathrm{~mm}$ of left ventricular hypertrophy (LVH), 19-20 increased of sodium removal and 21-22 improved fluid status [21]. The study of Roszkowska-Blaim, et al. 2013, discussed the consequences of loss of residual renal function on patients health and they suggested that, RRF loss may result in suboptimal dialysis adequacy, necessitating changes in the PD protocol such as increasing the amount of dialysate fluid used, including high osmolarity fluids, and in some cases combining PD with hemodialysis or switching from PD to an alternative modality of renal replacement therapy [22].

Patients post initiation on PD experienced good wellbeing indicated by clinical and physical features during the first period on PD. Our study reported improvement of the following laboratory investigations improved post PD; Hb, RBC, WBC, Hematocrit, platelet, Urea, creatinine, $\mathrm{GFR}, \mathrm{Ca}^{++}$, phosphate, $\mathrm{PTH}$ and $\mathrm{Na}^{+}$. The study of Jung, et al. 2019 [23] aimed to compare Health-Related Quality of Life (HRQOL) over time in patients initiating hemodialys is (HD) or Peritoneal Dialysis (PD), reported that PD shows better HRQOL during the initial period after dialysis even after adjusting for clinical and socioeconomic characteristics, and the effect lasts up to two years [23]. Another study done in Belgium in 1983 [24], investigated the anemia on CAPD, the study distributed the participants to three groups depends on their periods on PD, from 1 to 6 months, from 6 to 12 months and third group from 12 to 24 months, concluded that CAPD results in an early improvement of the uremic anemia, as demonstrated by a significant increase in red cell mass. However, the changes in routine hematological parameters are also profoundly influenced by an important decrease in circulating plasma volume occurring with this form of therapy.

Serum potassium was reduced by $12.5 \%$ post PD in our patients, which possibly was possibly achieved by both daily PD therapy and dietary restriction. In a study done by $\mathrm{Xu}$, et al. 2014 [25], a total of 886 incident PD patients were enrolled, with 248 patients (27.9\%) presented with hypokalemia (serum potassium $<3.5 \mathrm{mEq} / \mathrm{L}$ ). During a median follow-up of 31 months (range: 0.5-81.0 months), adjusted all-cause mortality Hazard Ratio (HR) and 95\% Confidence Interval $(\mathrm{Cl})$ for baseline serum potassium of $<3.0,3.0$ to $<3.5,3.5$ to $<4.0,4.5$ to $<5.0$, and $\geq 5.0 \mathrm{mEq} / \mathrm{L}$, compared with 4.0 to $<4.5$, were $1.79(1.02-3.14), 1.15(0.72-1.86), 1.31$ (0.82-2.08), $1.33(0.71-2.48), 1.28(0.53-3.10)$, respectively [25].

HBA1C and serum Albumin been affected negatively by peritoneal dialysis as approved by our study. Participants having normal serum HBA1C level been reduced from $54.1 \%$ before PD to $43.5 \%$ post initiation on PD. This can be explained by dietary intake or glucose reabsorption from PD fluid. The study of Coelho, et al. 2014 reviewed HBA1C with peritoneal dialysis and reported that in the PD population, there was a positive correlation between blood glucose level and peritoneal dialysis that contribute to changes in $\mathrm{HbA1c}$, although weaker due to the influence of other factors [26]. Furthermore, the daily glucose load of PD patients was conditioned not only by the diet but also by the potential reabsorption of glucose peritoneal fluids used as dialysate $[20,26]$. In PD, continuous glucose loading from the conventional dialysis solution may contribute to the increased cardiovascular risk that is a major cause of death in ESRD patients [27].

Peritoneal membrane prognosis is independent of baseline transport characteristics. Intrinsic fast transport and low UF are reversible conditions when peritonitis and high glucose exposure are avoided during the early dialysis period. Icodextrin helps in glucose avoidance and is associated with peritoneal protection [28]. The type of peritoneal membrane has a role on glucose absorption and fluid retention. Fast transporter membrane has the character of the fast dissipation of glucose from the peritoneal cavity, with negative ultrafiltration in dwells with $1.36 \%$ glucose longer than $180 \mathrm{~min}$. In this study the majority (40.4\%) of patients were having high average membrane and $9.6 \%$ had high transport membrane [28].

Hypoalbuminemia among our PD patients increased to $25.6 \%$ after starting on the peritoneal dialysis therapy. There are some factors contributed to the loss of albumin such as solute transport through diffusion process during peritoneal dialysis therapy or it could be the less consumption of proteins by the patients and/or the fluid overload status of the patients. A study done by Margetts, et al. suggested that low albumin is associated with high transport membrane and poor outcomes associated with low serum albumin and higher peritoneal membrane transport might be explained by other underlying factors. The contribution of inflammation, malnutrition, and fluid overload requires further study [29].

In this study, high triglyceride level increased among patients on PD by $4 \%$. The study by Natalia Stepanova 2019 found a strong association between lipid profile and PD adequacy parameters and it also found a significantly higher all-cause PD technique failure rate in patients with atherogenic dyslipidemia at baseline [30]. Moreover, the higher cause of this failure is explained by present of other factors such as longer PD duration, a decrease in RRF, and glucose absorption from the dialysis fluid.

World-wide, each country must have a specialized peritoneal dialysis nurse coordinator at each hospital and at regional areas of the country [31,32]. Having a nurse led program, utilizing a clinical pathway that runs in all clinics throughout the country is of utmost importance to improve the health related quality of life $[33,34]$. The program should involve a routine surveillance of all ESKD patients and a direct referral of cases 
to the nurse PD coordinator clinics $[32,35]$. This would lead to the development of many actions planned in relation to permanent PD dialysis-catheter care improvement.

There is an excellent health system in Oman, and it has been appraised highly by the World Health Organization $[4,5]$. Oman and its region at large have major issues with PD accessibility and management. There is a very urgent need to study the currently existing disparities for dialysis access. Initially, the survey should just focus to assess the current infrastructure that exist throughout the country including coordinators, dedicated access surgeons or interventionists. There is possibly a need for a wide-spread survey of PD services among all units with their responses being completely anonymized, to encourage everyone to participate and to therefore help us to be at a better position to understand the current existing gaps.

There is a need to collect PD access data from each individual Renal center throughout the country. This will enable us to understand the reasons behind the high failure for PD enrollment. There are many reasons, to mention a few such as patient refusal, late patient referral, poor patient selection, inadequate surgery service, lack of monitoring, poor surveillance, poor interventional services and insufficient education of both patients and clinicians. Building a multidisciplinary PD access team in each region, country or city is an essential starting step. This team requires a PD coordinator and an interventionist. The interventionist does not have to be an interventional nephrologist but can be a surgeon or an interventional radiologist. This should be supported with a surveillance and quality assurance program.

There is a need to provide a curriculum for the PD therapy coordinator that meticulously explains the PD program aims, methods, endpoints, surveillance, quality assurance and so on. There is also a need to have regular training courses for the coordinators, as well as various members of the PD therapy team. Collaboration with the International Societies of Nephrology and Peritoneal Dialysis is an important corner of the PD therapy/ access team for updates, follow ups of successes, challenges and to help with training and guidance.

The outcome of a PD therapy is reasonably very successful, both clinical and laboratory outcomes in ESKD. It is of great importance to have a multi-disciplinary approach including nurses, nephrologists, surgeons and intervention radiologists in ensuring an optimal PD therapy. A specialized-PD nurse coordinator is a significant contribution to any department to improve the success rates of PD among our population, to improve first longterm PD strategy and to improve the nurse led PD therapy surveillance program.

PD therapy coordinators should have regular meetings to discuss PD therapy issues and to regularly collect data for dialysis events including catheter related infections, dedicated and or access intervention time and the prevalence of cases in the hospital units. There is a regional need to cooperate, discuss, share data and experiences to promote better PD therapy care in our region. This will create a platform where professionals from all related PD-disciplines in the region could come together and share ideas and expertise on how to improve this aspect of PD-dialysis care with solutions which are more relevant to the region that share similar demography and patient characteristics.

\section{Conclusion}

PD service utilization is increasing as time pass on in Oman because of its advantages and restrains with hemodialysis. It gives higher chance for freedom, dependency and share responsibilities compared with hemodialysis. Moreover, it releases overwhelmed on hemodialysis centers. There was an almost equal gender distribution with young age and almost half have diabetes and $90 \%$ with high blood pressure. Most people utilized APD as a modality choice compared to CAPD. PD data showed improved on patients' wellbeing and quality of life over a year period of PD therapy. Most laboratory data and blood pressure reading improved, but there was a raised in some of blood investigation, such as, glycated hemoglobin and drop in serum albumin level.

It is recommended to work hardly on PD Continuous Quality Improvement (CQI) by identifying problems, develops solutions, monitor clinical outcomes and evaluates results in a clear manner in order to improve patients care in order to develop PD program in Oman.

\section{Funding}

None.

\section{Conflict of Interest}

Authors declare no conflict of interest.

\section{References}

1. Levey AS, Eckardt KU, Tsukamoto Y, Levin A, Coresh J, et al. (2005) Definition and classification of chronic kidney disease: a position statement from Kidney Disease: Improving Global Outcomes (KDIGO). Kidney Int 67: 2089-2100.

2. Neuen BL, Chadban SJ, Demaio AR, Johnson DW, Perkovic $V$ (2017) Chronic kidney disease and the global NCDs agenda. BMJ Glob Health 2: 000380.

3. (2020) Global, regional, and national burden of chronic kidney disease, 1990-2017: A systematic analysis for the Global Burden of Disease Study 2017. Lancet 395: 709733.

4. Al Salmi I, Hannawi S (2016) The World Health Report -Health systems Empowering Citizens and Improving Performance. Research on Humanities and Social Sciences 6: 6.

5. Al Salmi I, Hannawi S (2018) Health Workforce in the Sultanate of Oman: Improving performance and the Health System. J Int Med Pat Care 1: 6. 
6. Al Alawi IH, AI Salmi I, AI Mawali A, Sayer JA (2017) Kidney Disease in Oman: a View of the Current and Future Landscapes. Iran J Kidney Dis 11: 263-270.

7. Al Majarfi, Al Salmi, AM Metry, F. Al Ismaili, A. Hola, et al. (2018) Epidemiology of Patients at Initial Treatment with Hemodialysis. ARC Journal of Nephrology 3: 6.

8. Al Rahbi F, Al Salmi I (2017) Commercial Kidney Transplantation: Attitude, Knowledge, Perception, and Experience of Recipients. Kidney Int Rep 2: 626-633.

9. Al Salmi I, Metry AM, Al Ismaili F, Hola A, Al Riyami M, et al. (2018) Transplant tourism and invasive fungal infection. Int J Infect Dis 69: 120-129.

10. Alshaaili K, Salmi IA, Metry A, AI Ismail F, Hola A, et al. (2018) The Epidemiology of Hemolytic Uremic Syndrome: Clinical Presentation, Laboratory Findings, Management and Outcomes. Int J Hematol Blo Dis 3: 6.

11. Al Ismaili F, AI Salmi I, AI Maimani Y, Metry AM, Al Marhoobi $\mathrm{H}$, et al. (2017) Epidemiological Transition of End-Stage Kidney Disease in Oman. Kidney Int Rep 2: 27-35.

12. Blake PG (2001) Integrated end-stage renal disease care: the role of peritoneal dialysis. Nephrol Dial Transplant 5 : 61-66.

13. Al Alawi I, Al Salmi I, Al Mawali A, Al Maimani Y, Sayer JA (2017) End-Stage Kidney Failure in Oman: An Analysis of Registry Data with an Emphasis on Congenital and Inherited Renal Diseases. Int J Nephrol 2017: 6403985.

14. Najafi I, Alatab S, Atabak S, Majelan NN, Sanadgol H, et al (2014) Seventeen years' experience of peritoneal dialysis in Iran: first official report of the Iranian peritoneal dialysis registry. Peri Dial Int 34: 636-642.

15. Al Wakeel JS, Al Ghonaim MA, Aldohayan A, Usama S, Al Obaili S, et al. (2018) Appraising the outcome and complications of peritoneal dialysis patients in self-care peritoneal dialysis and assisted peritoneal dialysis: A 5-year review of a single Saudi center. Saudi J Kidney Dis Transpl 29: 71-80.

16. Al Rahbi N ASI, Ismaili M, Al Rawahi N, Karkar A (2020) Comparison of $2 \%$ Chlorhexidine and $70 \%$ Alcohol Swab Versus 10\% Povidone- lodine Solution in Preventing Peritoneal Dialysis Exit Site Infection. J Nephrol Renal Ther 6: 036.

17. Prasad N, Sinha A, Gupta A, Sharma RK, Bhadauria D, et al. (2014) Effect of body mass index on outcomes of peritoneal dialysis patients in India. Perit Dial Int 34: 399-408.

18. Kiran VR, Zhu TY, Yip T, Lui SL, Lo WK (2014) Body mass index and mortality risk in Asian peritoneal dialysis patients in Hong Kong-impact of diabetes and cardiovascular disease status. Perit Dial Int 34: 390-398.

19. Stern A, Sachdeva S, Kapoor R, Singh J, Sachdeva S (2014) High blood pressure in dialysis patients: cause, pathophysiology, influence on morbidity, mortality and management. J Clin Diagn Res 8: ME01-ME4.

20. Sarafidis PA, Persu A, Agarwal R, Burnier M, de Leeuw $P$, et al. (2017) Hypertension in dialysis patients: a consensus document by the European Renal and Cardiovascular Medicine (EURECA-m) working group of the European Renal Association-European Dialysis and Transplant Association (ERA-EDTA) and the Hypertension and the Kidney working group of the European Society of Hypertension (ESH). J Hypertens 32: 657-676.

21. Marrón $B$, Remón $C$, Pérez-Fontán $M$, Quirós $P$, Ortíz $A$ (2008) Benefits of preserving residual renal function in peritoneal dialysis. Kidney Int Suppl 2008: 42-51.
22. Roszkowska-Blaim M, Skrzypczyk P (2013) Residual renal function in children treated with chronic peritoneal dialysis. The Scientific World Journal 2013: 154537.

23. Jung HY, Jeon Y, Park Y, Kim YS, Kang SW, et al. (2019) Better Quality of Life of Peritoneal Dialysis compared to Hemodialysis over a Two-year Period after Dialysis Initiation. Scientific Reports 9: 10266.

24. De Paepe MB, Schelstraete $\mathrm{KH}$, Ringoir SM, Lameire $\mathrm{NH}$ (1983) Influence of continuous ambulatory peritoneal dialysis on the anemia of endstage renal disease. Kidney Int 23: $744-748$

25. Xu Q, Xu F, Fan L, Xiong L, Li H, et al. (2014) Serum potassium levels and its variability in incident peritoneal dialysis patients: associations with mortality. PLoS One 9: 86750.

26. Coelho S, Rodrigues A (2014) Hemoglobin A1c in patients on peritoneal dialysis: how should we interpret it? Ther Apher Dial 18: 375-382.

27. Fernández-Reyes MJ, Bajo MA, Del Peso G, Ossorio M, Díaz R, et al. (2012) The influence of initial peritoneal transport characteristics, inflammation, and high glucose exposure on prognosis for peritoneal membrane function. Perit Dial Int 32: 636-644.

28. van Biesen W, Heimburger O, Krediet R, Rippe B, La Milia V, et al. (2010) Evaluation of peritoneal membrane characteristics: clinical advice for prescription management by the ERBP working group. Nephrol Dial Transplant 25: 20522062.

29. Margetts PJ, McMullin JP, Rabbat CG, Churchill DN (2000) Peritoneal membrane transport and hypoalbuminemia: cause or effect? Perit Dial Int 20: 14-18.

30. Stepanova N, Burdeyna O (2019) Association between Dyslipidemia and Peritoneal Dialysis Technique Survival. Open Access Maced J Med Sci 7: 2467-2473.

31. Sola L, Levin NW, Johnson DW, Pecoits-Filho R, Aljubori $\mathrm{HM}$, et al. (2020) Development of a framework for minimum and optimal safety and quality standards for hemodialysis and peritoneal dialysis. Kidney Int Suppl (2011) 10: 55-62.

32. Yang C-W, Harris DCH, Luyckx VA, Nangaku M, Hou FF, et al. (2020) Global case studies for chronic kidney disease/ end-stage kidney disease care. Kidney Int Suppl (2011) 10: 24-48.

33. Al-Rajhi W, Al Salmi I (2020) Quality Of Life And Health Related Quality Of Life Among End-Stage Kidney Disease Patients: Methodology Assessment. J Nephrol Renal Ther 6: 23.

34. Al-Rajhi W, Al Salmi I (2020) Quality of Life and End Stage Kidney Disease: Conceptual and Theoretical Issues. J Nephrol Renal Ther 6: 7.

35. Chow SK, Wong FK (2010) Health-related quality of life in patients undergoing peritoneal dialysis: effects of a nurseled case management programme. J Adv Nurs 66: 17801792. 